\title{
INSTANTANEOUS STATES OF MARKOV PROCESSES
}

\author{
BY \\ GERALD SMITH
}

1. This paper answers a question suggested by Professor Kai Lai Chung concerning an instantaneous state of a countable state, continuous time, stationary Markov process. A countable state, continuous time, stationary Markov process is defined by its probability transition functions $p_{i j}(t), i, j=1,2,3, \cdots, t \geqq 0$, which satisfy the equations

$$
\begin{aligned}
p_{i j}(t) & \geqq 0, \quad \sum_{j=1}^{\infty} p_{i j}(t)=1, \\
\sum_{k=1}^{\infty} p_{i k}(s) p_{k j}(t) & =p_{i j}(s+t) .
\end{aligned}
$$

$p_{i j}(t)$ is interpreted to be the probability that the process will be in state $j$ at time $s+t$ given that it is in state $i$ at time $s$.

An additional condition which is usually imposed on the $p_{i j}(t)$, and which we "will impose, is that $\lim _{t \rightarrow 0} p_{i j}(t)=1$. This implies (see Chung [1]) the continuity of all the $p_{i j}(t)$, and the existence of all $p_{i j}^{\prime}(t)$, which may be $-\infty$ for $i=j, t=0$. Letting $p_{i j}^{\prime}(0)=q_{i j}, i \neq j$, and $p_{i i}^{\prime}(0)=-q_{i}$, we have $0 \leqq q_{i j}<\infty$ and $0 \leqq q_{i} \leqq \infty$. In the case $q_{i}=\infty$ we say $i$ is an instantaneous state. An equivalent definition (see Chung) of an instantaneous state is a state which the process cannot remain in for any positive time interval.

In $\S 2$ an example is presented of a Markov process with $\lim \sup _{t \rightarrow 0} p_{i i}^{\prime}(t)=+\infty$ for an instantaneous state $i$, answering negatively the question of whether $\lim _{t \rightarrow 0} p_{i i}^{\prime}(t)=-\infty$ always if $i$ is instantaneous.

2. A Markov process of a certain type, containing exactly one instantaneous state and a countable number of stable states will first be constructed, by the probabilistic construction of its sample functions.

Consider as in Chung $[1$, p. 255], a sequence of independent Poisson processes, all starting at time $t=0$, with parameter $\lambda_{i}$ for the $i$ th process, where $\sum_{i=1}^{\infty} \lambda_{i}=\infty$. As in Chung, assign a different color to each process. Let all the jumps of a given process be denoted by marks of the assigned color at the points of the jumps. Then superimpose all the processes with their different colored marks, with $t=0$ coinciding for all of them.

Received by the editors November 1, 1962. 
Definition 1. $F_{c}$ is a distribution function defined by $F_{c}(x)=0$ for $x<0$, and $F_{c}(x)=1-e^{-c x}$ for $x \geqq 0$.

Choose for each $i$ an $a_{i}>0$ so that $\sum_{i=1}^{\infty}\left(a_{i}\right)^{-1}<\infty$ and for each mark of the $i$ th color associate an ordered pair of numbers, each number of the pair being chosen probabilistically and independently from the distribution function $F_{\lambda_{i} a_{i}}$. Do this for all $i$, choosing the numbers independently for each mark. The result shall be called an $L$-process. More formally, we have

Definition 2. Let $\lambda_{i}>0, a_{i}>0, i=1,2, \cdots$. We shall call the set $\left(\omega,\left\{c_{j}^{(1)}\right\},\left\{d_{j}^{(1)}\right\},\left\{c_{j}^{(2)}\right\},\left\{d_{j}^{(2)}\right\}, \cdots\right)$ a sample function with assigned values of an an $L$-process if $\omega$ is a sample function defined on $t \geqq 0$ taking values $\gamma, 1,2, \cdots$, such that for all $t>0$ and integers $i$ the set of $s \geqq 0$ such that $\omega(s)=i$ and $s<t$ consists of at most a finite number of points, and the $\left\{c_{j}^{(i)}\right\}$ and $\left\{d_{j}^{(i)}\right\}$ are sequences of positive numbers for each integer $i$. A $\left\{\left\{\lambda_{i}\right\} ;\left\{a_{i}\right\}\right\}$, or more briefly, a $\left\{\lambda_{i} ; a_{i}\right\}$ $L$-process is a probability space of sample functions of assigned values of an $L$-process with the probabilistic construction as follows. Let $X_{\alpha_{i}}^{(j)}, X_{\beta_{1}}^{(j)}$, and $Y_{i}^{(j)}, i, j=1,2, \cdots$, be independent random variables, $X_{\alpha_{i}}^{(j)}$ and $X_{\beta_{i}}^{(j)}$ having distribution function $F_{\lambda_{i} a_{i}}$ for all $j$, and $Y_{i}^{(j)}$ having distribution function $F_{\lambda_{i}}$ for all $j$. Choose a point of the space of random variables. Associate with this point a sample function with assigned values of an $L$-process with $\omega(t)=i$ if $\sum_{j=1}^{n} Y_{i}^{(j)}=t$ for $i$ and some $n, \omega(t)=\gamma$ otherwise, and $\left\{c_{j}^{(i)}\right\}=\left\{X_{\alpha_{i}}^{(j)}\right\},\left\{d_{j}^{(i)}\right\}=\left\{X_{\beta_{i}}^{(j)}\right\}$. (Note that with probability one $\omega(t)$ can equal at most one $i$ for each $t$, so the $\omega$ is well-defined.) If $\omega(t)=i$ and $\sum_{j=1}^{n} Y_{i}^{(j)}=t$ we say $\omega$ has an $n$th mark of the $i$ th color at $t$, and we say the ordered pair $\left(X_{\alpha_{i}}^{(j)}, X_{\beta_{t}}^{(j)}\right)$ is assigned to this mark. We set $\operatorname{Prob}\{g(A)\}=\operatorname{Prob}\{A\}$, where $A$ is any measurable set in the space of random variables and $g(A)$ is the set of sample functions of assigned values associated with all members of $A$.

Definition 3. $S_{i}(t)$ is a function defined on sample functions with assigned values of an $L$-process such that $S_{i}(t)\left(\omega,\left\{c_{j}^{(1)}\right\}, \cdots\right)=\sum_{j=1}^{n}\left(c_{j}^{(i)}+d_{j}^{(i)}\right)$, where $n$ is such that $\sum_{j=1}^{n} Y_{i}^{(j)}<t \leqq \sum_{j=1}^{n+1} Y_{i}^{(j)}$.

THEOREM 1. With probability one a sample function with assigned values of an $L$-process will not have $\omega(s)=\gamma$ for all $s \in\left(t, t^{\prime}\right)$ for some $t^{\prime}>t$.

Proof. For any $t^{\prime}>t$ we have, due to the independence of the $X_{\alpha_{i}}^{(j)}, X_{\beta_{i}}^{(j)}$, and $Y_{i}^{(j)}$, the probability of no marks of the $i$ th color in $\left(t, t^{\prime}\right)$ equaling $e^{-\lambda_{i}\left(t^{\prime}-t\right)}$, and the probability of no marks at all in $\left(t, t^{\prime}\right)$ equaling

$$
\prod_{i=1}^{\infty} e^{-\lambda_{i}\left(t^{\prime}-t\right)}=e^{-\Sigma \lambda_{i}\left(t^{\prime}-t\right)}=e^{-\infty}=0 .
$$

Applying this to $t, t^{\prime}$ equaling each rational the result follows.

THEOREM 2. With probability one for any $t>0 \sum_{i=1}^{\infty} S_{i}(t)\left(\omega,\left\{c_{j}^{(1)}\right\}, \cdots\right)$ is finite for any L-process. 
Proof. Let $t>0$. Since $1 / \lambda_{i} a_{i}$ equals the expectation of each $c_{j}^{(i)}$ and $d_{j}^{(i)}$ and $\lambda_{i} \Delta s$ equals the probability of an $i$ th colored mark occurring in an interval of length $\Delta s$ for small $\Delta s$, we have

Then

$$
E\left(S_{i}(t)\right)=\int_{0}^{t} \lambda_{i} \frac{2}{\lambda_{i} a_{i}} d s=\frac{2 t}{a_{i}} .
$$

$$
\sum_{i=1}^{\infty} E\left(S_{i}(t)\right)=\sum_{i=1}^{\infty} \frac{2 t}{a_{i}}<\infty
$$

and hence $E\left(\sum_{i=1}^{\infty} S_{i}(t)\right)<\infty$ by the monotone convergence theorem. Letting $t$ tend to infinity through a denumerable sequence, the result follows.

Consequently an $L$-process may be converted to a Markov process with one instantaneous state and a denumerable number of stable states $\left\{\alpha_{i}\right\},\left\{\beta_{i}\right\}$, $i=1,2,3, \cdots$ as follows. Replace each mark occurring in the $\omega$ of a sample function with assigned values by two intervals of lengths equal to the two numbers associated with the mark, fixing the point $t=0$ and shifting the rest of the sample function to the right to make room for the new intervals. More precisely, if a a mark occuring at time $t$ of the original construction has the pair $(a, b)$ associated with it, then on the new axis place an interval of length $a$ with its beginning at time $t+\sum\left(a_{\eta}+b_{\eta}\right)$, where the sum is taken over all pairs $\left(a_{\eta}, b_{\eta}\right)$ associated with marks occuring before $t$ in the superimposed process. Place an interval of length $b$ such that it begins at time $t+a+\sum\left(a_{\eta}+b_{\eta}\right)$ with the same sum $\Sigma\left(a_{\eta}+b_{\eta}\right)$ as for $a$. Let the first number of the ordered pairs associated with marks of the $i$ th color represent lengths of the stable state $\alpha_{i}$, and the second the stable state $\beta_{i}$. Let the time not taken up by the stable states represent the occurrence of the state $\gamma$, which is obviously instantaneous since it lasts for no interval. By the nature of the construction the process is Markovian. Formally, we have

Definition 4. Let $\lambda_{i}>0, a_{i}>0, \quad i=1,2, \cdots, \sum_{i=1}^{\infty} \lambda_{i}=\infty, \sum_{i=1}^{\infty}\left(a_{i}\right)^{-1}<\infty$. A $\left\{\left\{\lambda_{i}\right\} ;\left\{a_{i}\right\}\right\}$, or more briefly, a $\left\{\lambda_{i} ; a_{i}\right\} M$-process is a process defined on $t \geqq 0$ whose sample functions $\omega$ are probabilistically constructed as follows. Let $X_{\alpha_{i}}^{(j)}, X_{\beta_{i}}^{(j)}$, and $Y_{i}^{(j)}, i, j=1,2, \cdots$ be independent random variables, with $X_{\alpha_{i}}^{(j)}$ and $X_{\beta_{i}}^{(j)}$ having the distribution function $F_{\lambda_{i} a_{i}}$, all $j$, and $Y_{i}^{(j)}$ having the distribution function $F_{\lambda_{i}}$, all $j . \omega(t)=\alpha_{l}$ if for some $0<s<t^{\prime}<t$

$$
\sum_{i=1}^{\infty} \sum_{n=1}^{k(j i)}\left[X_{\alpha_{i}}^{(n)}+X_{\beta_{i}}^{(n)}\right]+s=t^{\prime}
$$

where $k(j, i)$ is the unique integer such that $\sum_{n=1}^{k(j, i)} Y_{i}^{(n)} \leqq s<\sum_{n=1}^{k(j, i)+1} Y_{i}^{(n)}$, and $\sum_{n=1}^{k(j, l)} Y_{l}^{(n)}=s$ and

$$
X_{\alpha_{l}}^{k(J, l)} \geqq t-t^{\prime}
$$

(Note that with probability one there will not be integers $m, n, p$, and $q$ such that 
$m \neq n$, and $\sum_{j=1}^{p} Y_{m}^{(j)}=\sum_{j=1}^{q} Y_{n}^{(j)}=s$ for some $\left.s.\right) \omega(t)=\beta_{l}$ if the above equations hold with $X_{\alpha_{l}}^{k(j, l)}<t-t^{\prime} \leqq X_{\alpha_{l}}^{k(j, l)}+X_{\beta_{l}}^{k(j, l)}$ replacing $\left(^{*}\right) . \omega(t)=\gamma$ elsewhere.

The desired process is of this type. The parameters will be chosen so that $\lim \sup _{t \rightarrow 0} p_{\gamma \gamma}^{\prime}(t)=+\infty$. Before this can be done, however, some preliminary definitions and theorems are needed.

Definition 5. Let $\lambda>0, a>0$. We shall call the set $\left(\omega,\left\{c_{i}\right\},\left\{d_{i}\right\}\right)$ a sample function with assigned values of an $S$-process if $\omega$ is a sample function defined on $t \geqq 0$ taking values $\gamma$ and 1 such that for all $t>0$ the set of $s \geqq 0$ such that $\omega(s)=1$ and $s<t$ consists of at most a finite number of points, and $\left\{c_{i}\right\}$ and $\left\{d_{i}\right\}$ are sequences of positive numbers. A $(\lambda, a) S$-process is a probability space of sample functions of assigned values of an $S$-process with the probability measure determined as follows. Let $X_{\alpha}^{(j)}, X_{\beta}^{(j)}$, and $Y^{(j)}, j=1,2, \cdots$ be independent random variables, all $X_{\alpha}^{(j)}$ and $X_{\beta}^{(j)}$ having distribution function $F_{\lambda a}$, and all $Y^{(j)}$ having distribution function $F_{\lambda}$. Choose a point of the space of random variables. Associate with this point a sample function with assigned values of an $S$-process such that $\omega(t)=1$ if $\sum_{j=1}^{n} Y^{(j)}=t$ for some $n$, and $\omega(t)=\gamma$ otherwise, and $\left\{c_{i}\right\}=\left\{X_{\alpha}^{(i)}\right\},\left\{d_{i}\right\}=\left\{X_{\beta}^{(i)}\right\}$. We set $\operatorname{Prob}\{g(A)\}=\operatorname{Prob}\{A\}$, where $A$ is any measurable set in the space of random variables and $g(A)$ is the set of sample functions of assigned values associated with all members of $A$. We shall say the ordered pair $\left(X_{\alpha}^{(i)}, X_{\beta}^{(i)}\right)$ is assigned to the $i$ th mark of $\omega$.

Definition 6. Let $\lambda>0, a>0$. $\mathrm{A}(\lambda, a) T$-process is a process defined on $t \geqq 0$ whose sample functions $\omega$ are probabilistically constructed as follows. Let $X_{\alpha}^{(j)}, X_{\beta}^{(j)}$, and $Y^{(j)}, j=1,2, \cdots$ be independent random variables, with all $X_{\alpha}^{(j)}$ and $X_{\beta}^{(j)}$ having distribution function $F_{\lambda a}$ and all $Y^{(j)}$ having distribution function $F_{\lambda} . \omega(t)=\gamma$ if for some $n$

$$
\sum_{j=1}^{n-1}\left(X_{\alpha}^{(j)}+X_{\beta}^{(j)}+Y^{(j)}\right) \leqq t<\sum_{j=1}^{n-1}\left(X_{\alpha}^{(j)}+X_{\beta}^{(j)}+Y^{(j)}\right)+Y^{(n)}
$$

$\omega(t)=\alpha$ if for some $n$

$$
\sum_{j=1}^{n-1}\left(X_{\alpha}^{(j)}+X_{\beta}^{(j)}+Y^{(j)}\right)+Y^{n} \leqq t<\sum_{j=1}^{n-1}\left(X_{\alpha}^{(j)}+X_{\beta}^{(j)}+Y^{(j)}\right)+X_{\alpha}^{(n)}+Y^{(n)} .
$$

$\omega(t)=\beta$ otherwise. Whenever an $M$-process with parameters $\left\{\left\{\lambda_{i}\right\} ;\left\{a_{i}\right\}\right\}$ is being discussed, we shall understand by an $S_{k}$-process, for fixed $k$, a $\left(\lambda_{k}, a_{k}\right) S$-process, and by a $T_{k}$-process a $\left(\lambda_{k}, a_{k}\right) T$-process.

Thus a $(\lambda, a) T$-process is a three state Markov process with nonabsorbing states $\gamma, \alpha$, and $\beta$, which starts in $\gamma$ at $t=0$ and moves cyclicly in the order $\gamma \rightarrow \alpha \rightarrow \beta \rightarrow \gamma \rightarrow \alpha \rightarrow \cdots$, etc., and which has $q_{\gamma}=\lambda, q_{\alpha}=q_{\beta}=\lambda a$. Note that if the marks of the $i$ th color in a given $L$-process were expanded to pairs of intervals as in the construction of an $M$-process, and all other marks were deleted, a $\left(\lambda_{i}, a_{i}\right)$ $T$-process would result. $\gamma$ retains its original role, and $\alpha_{i}$ and $\beta_{i}$ play the roles of 
the $\alpha$ and $\beta$ of the $T$-process. An $S$-process is to a $T$-process what an $L$-process is to an $M$-process. Also, an $L$-process may be considered to be the superposition of a countable number of independent $S$-processes. Analogously to $S_{i}(t)$, we have

Definition 7. $S(t), t \geqq 0$, is a function on sample functions with assigned values of an $S$-process such that $S(t)\left(\omega,\left\{c_{i}\right\},\left\{d_{i}\right\}\right)=\sum_{i=1}^{n}\left(c_{i}+d_{i}\right)$ where $n$ is such that $\sum_{j=1}^{n} Y^{(j)}<t \leqq \sum_{j=1}^{n+1} Y^{(j)}$.

Definition 8. A first process of one of the above types $(L, M, S$, or $T)$ is said to be finer than a second of the same type if each parameter of the first is at least as large as the corresponding parameter of the second (e.g. in an $L$ - or $M$-process $\lambda_{i}^{(1)} \geqq \lambda_{i}^{(2)}$ and $a_{i}^{(1)} \geqq a_{i}^{(2)}$ for all $i$, the superscripts referring to the first and second processes, respectively.) A parameter $\lambda,(a)$, is said to be finer than another parameter $\lambda^{\prime},\left(a^{\prime}\right)$, if $\lambda \geqq \lambda^{\prime},\left(a \geqq a^{\prime}\right)$.

Definition 9. An $L$-process ( $M$-process) with parameters $\left\{\lambda_{i} ; a_{i}\right\}$ is said to be $n$-finer than a second $L$-process ( $M$-process) with parameters $\left\{\lambda_{i}^{\prime} ; a_{i}^{\prime}\right\}$ if the first process is finer than the second and if $\lambda_{i}=\lambda_{i}^{\prime}$ and $a_{i}=a_{\imath}^{\prime}$ for all $i \leqq n$.

Since $M$ - and $T$-processes are stationary Markov processes, there exist transition probability functions between the states of either of these processes, and these functions will be used in Definitions 10 and 14 .

Definition 10. Let $t^{\prime}>t>0$. We shall say $\mathrm{DQ}\left(t^{\prime}, t\right) \geqq K$, for a $T$-process or $M$-process if $\left(p_{\gamma \gamma}\left(t^{\prime}\right)-p_{\gamma \gamma}(t)\right) /\left(t^{\prime}-t\right) \geqq K$. (DQ $\equiv$ difference quotient.)

Definition 11. We shall say $\mathrm{DQ}\left(t^{\prime}, t\right) \geqq K, n$-fixed, for an $M$-process if all $n$-finer $M$-processes have DQ $\left(t^{\prime}, t\right) \geqq K$.

Definition 12. Let $\eta>0$. We shall say $\mathrm{DQ}\left(t^{\prime}, t\right) \geqq K,(\varepsilon, \eta)$ protected, for a $T$-process if

$$
\begin{aligned}
& \frac{\operatorname{Prob}\left\{\omega(s)=\gamma \text { for all } s \in\left[t^{\prime}-\eta, t^{\prime}\right]\right\}}{t^{\prime}-t} \\
& \quad-\frac{\operatorname{Prob}\{\omega(s)=\gamma \text { for some } s \in[t-\eta, t]\}+\varepsilon}{t^{\prime}-t} \geqq K .
\end{aligned}
$$

(If $\eta>t$ then $t-\eta$ should be replaced by 0 .)

Definition 13. An $M$-process is pre $(\varepsilon, n, t)$ free if $n=1$ or if

$$
\operatorname{Prob}\left\{\sum_{i=1}^{n-1} S_{i}(t)>0\right\}<\frac{\varepsilon}{4} .
$$

Definition 14. An $M$-process is post $(\varepsilon, n, t, \eta)$ free if

and

$$
\operatorname{Prob}\left\{\sum_{i=n+1}^{\infty} S_{i}(t)>\eta\right\}<\frac{\varepsilon}{8},
$$

$$
\sum_{i=n+1}^{\infty}\left[p_{\gamma \alpha_{i}}\left(t^{\prime}\right)+p_{\gamma \beta_{i}}\left(t^{\prime}\right)\right]<\frac{\varepsilon}{8}
$$

for all $t^{\prime}$, and if these two equations also hold for all $n$-finer $M$-processes. 
The essential result allowing the desired process to be constructed is Lemma 1 of Theorem 3, giving the existence of a $t$ for certain $T$-processes such that $p_{\gamma \gamma}^{\prime}(t)>0$. Lemmas 2 and 3 of the same theorem allow changes in the $T$-process so that there exists $p_{\gamma \gamma}^{\prime}(t)$ as large as desired, for some $t$. For any integer $n$, an $M$ process would be a $T_{n}$-process if it were not for the interference of intervals $\alpha_{i}$ and $\beta_{i}, i \neq n$. Specifically, it could be a $T$-process having a large positive $p_{\gamma \gamma}^{\prime}(t)$. Although this interference exists, it can be made sufficiently small so that the desired result is still obtainable. Interference coming from those intervals with $i<n$ is made sufficiently small by making it pre $\left(\varepsilon, n, t^{\prime}\right)$ free, and interference coming from intervals with $i>n$ is made small by making it post $\left(\varepsilon, n, t^{\prime}, \eta\right)$ free. Then the $T_{n}$ process predominates so that $p_{\gamma \gamma}^{\prime}(t)$ is sufficiently large for a suitable $t$. This $T_{n}$-process predomination construction will be done in turn for $n=1,2,3, \cdots$ without subsequent constructions altering results already obtained. All steps will then be considered simultaneously, giving a well-defined process having the property $\limsup _{t \rightarrow 0} p_{\gamma \gamma}^{\prime}(t)=+\infty$.

The following theorems concern the decreasing of interference in a $T_{n}$-process.

THEOREM 3. Let an $M$-process have parameters $\left\{\lambda_{i} ; a_{i}\right\}$, let $T>0, K>0$ and let $n$ be a positive integer. There exist parameters $\lambda_{n}^{\prime}$ and $a_{n}^{\prime}$ finer than $\lambda_{n}$ and $a_{n}$, respectively, $\varepsilon>0, \eta>0, t$, and $t^{\prime}$ such that $T>t^{\prime}>t>0, \mathrm{DQ}\left(t^{\prime}, t\right) \geqq K$, $(\varepsilon, \eta)$ protected for the $\left(\lambda_{n}^{\prime}, a_{n}^{\prime}\right) T$-process, and such that the M-process which results from replacing $\lambda_{n}$ and $a_{n}$ by $\lambda_{n}^{\prime}$ and $a_{n}^{\prime}$, respectively is $\operatorname{pre}\left(\varepsilon, n, t^{\prime}\right)$ free.

This theorem will be proved by first proving six lemmas.

LemMA 1. Let the parameters $(\lambda, a)$ be given. There exist parameters $\left(\lambda, a^{\prime}\right)$ and $t>0$ such that $a^{\prime} \geqq a$ and $p_{\gamma \gamma}^{\prime}(t)>0$ for the $\left(\lambda, a^{\prime}\right) T$-process.

Proof. Employing the forward differential equation

$$
p_{i j}^{\prime}(t)=-p_{i j}(t) q_{j}+\sum_{k \neq j} p_{i k}(t) q_{k j}
$$

for Markov transition probability functions, we have

$$
\begin{aligned}
& p_{\gamma \gamma}^{\prime}(t)=-p_{\gamma \gamma}(t) \lambda+p_{\gamma \beta}(t) \lambda a^{\prime} \\
& p_{\gamma \alpha}^{\prime}(t)=-p_{\gamma \gamma}(t) \lambda a^{\prime}+p_{\gamma \gamma}(t) \lambda \\
& p_{\gamma \beta}^{\prime}(t)=-p_{\gamma \beta}(t) \lambda a^{\prime}+p_{\gamma \alpha}(t) \lambda a^{\prime} .
\end{aligned}
$$

By substitution we obtain

$$
p_{\gamma \gamma}^{\prime \prime \prime}(t)+\left(2 \lambda a^{\prime}+\lambda\right) p_{\gamma \gamma}^{\prime \prime}(t)+\left(2 \lambda^{2} a^{\prime}+\lambda^{2} a^{\prime 2}\right) p_{\gamma \gamma}^{\prime}(t)=0
$$


solving this for $p_{\gamma \gamma}^{\prime}(t)$, using the condition that $p_{\gamma \gamma}^{\prime}(t)$ be real and $p_{\gamma \gamma}^{\prime}(0)=-\lambda$, we obtain

$$
p_{\gamma \gamma}^{\prime}(t)=-\frac{\lambda}{2} e^{-\left(\lambda\left(2 a^{\prime}+1\right) / 2\right) t}\left[e^{\left(\lambda\left(\sqrt{ }\left(1-4 a^{\prime}\right)\right) / 2\right) t}+e^{-\left(\lambda\left(\sqrt{ }\left(1-4 a^{\prime}\right) / 2\right) t\right.}\right] .
$$

For $a^{\prime}>\frac{1}{4}$ the expression in brackets will be the sum of two complex conjugate circular functions and hence for any such $a^{\prime}$ there will be a $t>0$ for which their sum is negative and hence for which $p_{\gamma \gamma}^{\prime}(t)$ is positive.

From the definition of derivative it is obvious that there exist $t^{\prime}>t>0$ for the $\left(\lambda, a^{\prime}\right) T$-process for which $\mathrm{DQ}\left(t^{\prime}, t\right) \geqq d$ for some $d>0$.

LEMMA 2. If $p_{\gamma \gamma}(t)$ and $\bar{p}_{\gamma \gamma}(t)$ are the transition probability functions for $(\lambda, a)$ and $(\bar{\lambda}, a) T$-processes, respectively, then $p_{\gamma \gamma}(t / \lambda)=\bar{p}_{\gamma \gamma}(t / \bar{\lambda})$ for all $t$.

Proof. $\lambda$ and $\bar{\lambda}$ appear in the definitions of the $T$-processes merely as timescale parameters such that the above equation is true for all $t$.

LEMmA 3. If $\mathrm{DQ}\left(t^{\prime}, t\right) \geqq d \geqq 0$ for $a(\lambda, a)$ T-process, then $\mathrm{DQ}\left(\lambda t^{\prime} / \bar{\lambda}, \lambda t / \bar{\lambda}\right)$ $\geqq \bar{\lambda} d / \lambda$ for $a(\bar{\lambda}, a) T$-process.

Proof. This follows from Lemma 2 and consideration of the difference quotient.

LEMMA 4. If $\mathrm{DQ}\left(t^{\prime}, t\right) \geqq K+\delta$ for a $T$-process and some $\delta>0$, then there is an $\varepsilon>0$ and $\eta>0$ such that $\mathrm{DQ}\left(t^{\prime}, t\right) \geqq K,(\varepsilon, \eta)$ protected.

Proof. There exists an $\eta>0$ such that for the $T$-process

$$
p_{\gamma \gamma}\left(t^{\prime}\right)-\operatorname{Prob}\left\{\omega(s)=\gamma \text { for all } s \in\left[t^{\prime}-\eta, t^{\prime}\right]\right\} \leqq \frac{\delta}{3}\left(t^{\prime}-t\right)
$$

and

$$
\operatorname{Prob}\{\omega(s)=\gamma \text { for some } s \in[t-\eta, t]\}-p_{\gamma \gamma}(t) \leqq \frac{\delta}{3}\left(t^{\prime}-t\right)
$$

Choosing $\varepsilon=\delta\left(t^{\prime}-t\right) / 3$, the quotient in Definition 11 will then be at least

$$
\begin{gathered}
\frac{p_{\gamma \gamma}\left(t^{\prime}\right)-\frac{\delta}{3}\left(t^{\prime}-t\right)-p_{\gamma \gamma}(t)-\frac{\delta}{3}\left(t^{\prime}-t\right)-\frac{\delta}{3}\left(t^{\prime}-t\right)}{t^{\prime}-t} \\
=\frac{p_{\gamma \gamma}\left(t^{\prime}\right)-p_{\gamma \gamma}(t)}{t^{\prime}-t}-\delta \geqq K .
\end{gathered}
$$

LEMMA 5. If $\mathrm{DQ}\left(t^{\prime}, t\right) \geqq K,(\varepsilon, \eta)$ protected for $a(\lambda, a) T$-process, where $K>0$, then for any $\bar{\lambda} \geqq \lambda \mathrm{DQ}\left(\lambda t^{\prime} / \bar{\lambda}, \lambda t / \bar{\lambda}\right) \geqq K,(\varepsilon, \lambda \eta / \bar{\lambda})$ protected for $a(\bar{\lambda}, a)$ $T$-process.

Proof. Letting $\omega$ and $\bar{\omega}$ be sample functions for the respective $T$-processes, 


$$
\begin{aligned}
& \left(\frac{\lambda t^{\prime}}{\bar{\lambda}}-\frac{\lambda t}{\bar{\lambda}}\right)^{-1}\left(\operatorname{Prob}\left\{\bar{\omega}(s)=\gamma \text { for all } s \in\left(\frac{\lambda t^{\prime}}{\bar{\lambda}}-\frac{\lambda \eta}{\bar{\lambda}}, \frac{\lambda t^{\prime}}{\bar{\lambda}}\right)\right\}\right. \\
& \left.-\operatorname{Prob}\left\{\bar{\omega}(s)=\gamma \text { for some } s \in\left(\frac{\lambda t}{\bar{\lambda}}-\frac{\lambda \eta}{\bar{\lambda}}, \frac{\lambda t}{\bar{\lambda}}\right)\right\}-\varepsilon\right) \\
& =\left(\frac{\lambda t^{\prime}}{\bar{\lambda}}-\frac{\lambda t}{\bar{\lambda}}\right)^{-1}\left(\operatorname{Prob}\left\{\omega(s)=\gamma \text { for all } s \in\left(t^{\prime}-\eta, t^{\prime}\right)\right\}\right. \\
& -\operatorname{Prob}\{\omega(s)=\gamma \text { for some } s \in(t-\eta, t)\}-\varepsilon) \geqq K
\end{aligned}
$$

the last inequality resulting from $\bar{\lambda} \geqq \lambda$ and $\mathrm{DQ}\left(t^{\prime}, t\right) \geqq K,(\varepsilon, \eta)$ protected.

LEMMA 6. Let an $M$-process have parameters $\left\{\lambda_{i} ; a_{i}\right\}$, let $\varepsilon>0$, and $n$ be a positive integer. There exists a $t>0$ such that the $M$-process is pre $(\varepsilon, n, t)$ free.

Proof. If $n=1$ the lemma is trivial. If not, let $t_{i}>0$ be such that the probability of a mark of the $i$ th color occuring in $\left(0, t_{i}\right)$ in a $\left\{\lambda_{i} ; a_{i}\right\} L$-process is less than $\varepsilon / 4(n-1)$. Then let $t=\min _{i=1,2, \ldots, n-1} t_{i}$.

The theorem follows by first increasing $a_{n}$ to $a_{n}^{\prime}$ so that $p_{\gamma \gamma}^{\prime}(t)>0$ in the $T$ process for some $t$ by Lemma 1 , then increasing $\lambda_{n}$ so that $\mathrm{DQ}\left(t^{\prime}, t\right) \geqq K,(\varepsilon, \eta)$ protected for another $t, t^{\prime}$, and some $\varepsilon>0, \eta>0$ by the remark before Lemma 2, and Lemma 4 , and finally by increasing $\lambda_{n}$ more by Lemmas 5 and 6 so that the $M$-process is also pre $\left(\varepsilon, n, t^{\prime}\right)$ free for the $t^{\prime}$ in the difference quotient.

THEOREM 4. Let an $M$-process have parameters $\left\{\lambda_{i} ; a_{i}\right\}$, let $K>0, \varepsilon>0$, $\eta>0, t>0$, and let $n$ be a positive integer. There exists an $n$-finer $M$-process which is post $(\varepsilon, n, t, \eta)$ free.

This theorem will be proved by first proving four lemmas.

LEMmA 1. Let $\eta>0, \varepsilon>0, t>0$. There exists $a(\lambda, a) T$-process such that for all finer $T$-processes $S(t)$ of the related $S$-process with the same parameters will be less than $\eta$ with probability at least $1-\varepsilon$.

Proof. Choosing a pair of parameters $(\lambda, a)$ we see the expected length of $\gamma$ intervals is $1 / \lambda$. By the strong law of large numbers there exists $N_{1}$ such that with probability at least $1-\varepsilon / 3$ the average length of the first $n \gamma$ intervals will be greater than $1 / 2 \lambda$ for all $n>N_{1}$ and $\lambda$. Since the expected length of $\alpha$ and $\beta$ intervals is $1 / \lambda a$, there exists an $N_{2}$ such that except for probability at most $\varepsilon / 3$ the average length of the pairs of $\alpha$ and $\beta$ intervals, adding the length of an $\alpha$ interval to the length of the $\beta$ interval following it, is less than $3 / \lambda a$ for all $n>N_{2}, \lambda$, and $a$. There exists a $\lambda>0$ such that except for probability at most $\varepsilon / 3$ there will be at least $\max \left(N_{1}, N_{2}\right)$ marks appearing before $t$ in any $S$-process with parameters at least as fine as $(\lambda, a)$, for any $a$. Keep this $\lambda$ fixed and choose $a>6 t / \eta$. By the above, except for a set of probability at most $\varepsilon$ there will be at most $t(1 / 2 \lambda)^{-1}$ marks 
in $(0, t)$, and $S(t)$ will be at most $2 \lambda t(3 / \lambda a)<\eta$. By the nature of the proof it is apparant that all finer $T$-processes will also have this property.

Lemma 2. Let $\varepsilon>0, \eta>0, t>0$, and let $n$ be a positive integer. There exists an $M$-process with parameters $\left\{\lambda_{i} ; a_{i}\right\}$ such that for all finer processes $\operatorname{Prob}\left\{\sum_{i=n+1}^{\infty} S_{i}(t)>\eta\right\}<\varepsilon / 8$.

Proof. By Lemma 1 make $\left(\lambda_{i}, a_{i}\right), i \geqq n+1$, fine enough so

$$
\operatorname{Prob}\left\{S_{i}(t)>\frac{\eta}{2^{n+2-i}}\right\}<\frac{\varepsilon}{8 \times 2^{n+2-i}}
$$

and by addition Lemma 2 follows.

Lemma 3. Let $\varepsilon>0, t>0$. There exist parameters $(\lambda, a)$ such that for all $T$-processes at least that fine $\operatorname{Prob}\{\omega(t)=\alpha$ or $\beta\}<\varepsilon$.

Proof. Suppose $\omega\left(t^{\prime}\right)=\alpha$. There exists a $c_{1}>0$ such that the probability that $\omega$ remains entirely in $\alpha$ during $\left(t^{\prime}, t^{\prime}+c_{1} / 2 \lambda a\right)$ is less than $\varepsilon / 4$, whatever be $\lambda$ and $a$. Suppose $\omega\left(t^{\prime}\right)=\gamma$. There exists a $c_{2}>0$ such that the probability that $\omega$ remains in $\gamma$ during $\left(t^{\prime}, t^{\prime}+c_{2} / \lambda\right)$ is at least $1-\varepsilon / 4$, whatever be $\lambda$. Choose $a^{*}>c_{1} / c_{2}$. Then $c_{2} / \lambda>c_{1} / \lambda a^{*}$ for all $\lambda$. The parameters $\left(\lambda, a^{*}\right)$ meet the requirements of the lemma, for all $\lambda>0$. For first suppose $t-c_{2} / \lambda>0$. Then set $t^{\prime}=t-c_{2} / \lambda$. For $\omega(t)$ to equal $\alpha$, either $\omega\left(t^{\prime}\right)=\alpha$ and $\omega$ remains in $\alpha$ during $\left(t^{\prime}, t\right)$, an event of probability less than $\varepsilon / 4$, or $\omega\left(t^{\prime \prime}\right)=\gamma$ for some $t^{\prime \prime}$ in $\left(t^{\prime}, t\right)$, and then $\omega$ enters $\alpha$ before $t$, an event of probability less than $\varepsilon / 4$. Thus $\omega(t)=\alpha$ is an event of probability less than $\varepsilon / 2$. If $t \leqq c_{2} / \lambda$, then the event $\omega(t)=\alpha$ has probability less than $\varepsilon / 4$, hence less than $\varepsilon / 2$. This same argument can be applied to show for any $t \operatorname{Prob}\{\omega(t)=\beta\}<\varepsilon / 2$, and hence the lemma follows.

LEMMA 4. Let $\varepsilon>0$ and $n$ be a positive integer. There exists an M-process with parameters $\left\{\lambda_{i} ; a_{i}\right\}$ such that for all finer $M$-processes, and all $t>0$, $\operatorname{Prob}\left\{\omega(t)=\alpha_{i}\right.$ or $\beta_{i}$ for any $\left.i \geqq n+1\right\}<\varepsilon / 8$.

Proof. By Lemma 3 choose $\left(\lambda_{i}, a_{i}\right), i \geqq n+1$, so fine that Prob $\left\{\omega(t)=\alpha_{i}\right.$ or $\left.\beta_{i}\right\}$ $<\varepsilon / 8 \times 2^{n+2-i}$ for the $\left(\lambda_{i}, a_{i}\right) T$-process. Then this equation will also hold in the resulting $M$-process, as can be seen by the independence of the $S_{i}$ processes and considering the point $t-c_{2}^{(i)} / \lambda_{i}$ and proceeding as in the proof of Lemma 3.

Lemmas 2 and 4 give the theorem by definition.

TheOREM 5. Let an $M$-process have parameters $\left\{\lambda_{i} ; a_{i}\right\}$, let $K>0, \varepsilon>0$, $\eta>0, t^{\prime}>t>0$, and let $n$ be a positive integer. If the $\left(\lambda_{n}, a_{n}\right) T$-process has $\mathrm{DQ}\left(t^{\prime}, t\right) \geqq K,(\varepsilon, \eta)$ protected and if the $M$-process is pre $\left(\varepsilon, n, t^{\prime}\right)$ free and post $\left(\varepsilon, n, t^{\prime}, \eta\right)$ free, then $\mathrm{DQ}\left(t^{\prime}, t\right) \geqq K, n$-fixed.

Proof. Consider any $n$-finer $M$-process. Letting $\bar{p}$ and $\bar{\omega}$ be transition proba- 
bility and sample functions for this process, and $p$ and $\omega$ those for the $\left(\lambda_{n}, a_{n}\right)$ $T$-process, we have

$$
\begin{aligned}
p_{\gamma \gamma}\left(t^{\prime}\right)-\bar{p}_{\gamma \gamma}\left(t^{\prime}\right) \leqq & \operatorname{Prob}\left\{\omega\left(t^{\prime}\right)=\gamma \text { and } \omega(s) \neq \gamma \text { for some } s \in\left(t^{\prime}-\eta, t^{\prime}\right)\right\} \\
& +\operatorname{Prob}\left\{\sum_{i=n+1}^{\infty} S_{i}\left(t^{\prime}\right)>\eta\right\}+\sum_{i=n+1}^{\infty}\left[\bar{p}_{\gamma \alpha_{i}}\left(t^{\prime}\right)+\bar{p}_{\gamma \beta_{i}}\left(t^{\prime}\right)\right] \\
& +\operatorname{Prob}\left\{\sum_{i=1}^{n-1} S_{i}\left(t^{\prime}\right)>0\right\} \\
\leqq & \operatorname{Prob}\left\{\omega\left(t^{\prime}\right)=\gamma \text { and } \omega(s) \neq \gamma \text { for some } s \in\left(t^{\prime}-\eta, t^{\prime}\right)\right\}+\frac{\varepsilon}{2} .
\end{aligned}
$$

The various interference effects are brought to light in the above inequalities. The terms to the right of the first inequality are present because for an $\omega$ sample function with $\omega\left(t^{\prime}\right)=\gamma$ to be changed to an $\bar{\omega}$ sample function with $\bar{\omega}\left(t^{\prime}\right) \neq \gamma$ by interference with intervals with subscripts $i>n$, either $\omega(s)$ must not equal $\gamma$ everywhere in the interval $\left(t^{\prime}-\eta, t^{\prime}\right)$ and it must have this point pushed forward to $t^{\prime}$ by $\alpha_{i}$ and $\beta_{i}$ intervals $(i>n)$ of total length less than $\eta$, or it must have the sum of the $\alpha_{i}$ and $\beta_{i}$ intervals $(i>n)$ greater than $\eta$ to push an even farther back point $s$ with $\omega(s) \neq \gamma$ up to $t^{\prime}$, or an $\alpha_{i}$ or $\beta_{t}$ must itself cover $t^{\prime}$. The last term represents interference from the $i$ th coordinates with $i<n$.

From the above inequality

$$
\bar{p}_{\gamma \gamma}\left(t^{\prime}\right) \geqq \operatorname{Prob}\left\{\omega(s)=\gamma \text { for all } s \in\left(t^{\prime}-\eta, t^{\prime}\right)\right\}-\frac{\varepsilon}{2} .
$$

Also

$$
\begin{aligned}
\bar{p}_{\gamma \gamma}(t)-p_{\gamma \gamma}(t) \leqq & \operatorname{Prob}\{\omega(t) \neq \gamma \text { and } \omega(s)=\gamma \text { for some } s \in(t-\eta, t)\} \\
& +\operatorname{Prob}\left\{\sum_{t=n+1}^{\infty} S_{i}(t)>\eta\right\}+\operatorname{Prob}\left\{\sum_{i=1}^{n-1} S_{i}(t)>0\right\} \\
\leqq & \operatorname{Prob}\{\omega(t) \neq \gamma \text { and } \omega(s)=\gamma \text { for some } s \in(t-\eta, t)\}+\frac{\varepsilon}{2}
\end{aligned}
$$

Hence

$$
\bar{p}_{\gamma \gamma}(t) \leqq \operatorname{Prob}\{\omega(s)=\gamma \text { for some } s \in(t-\eta, t)\}+\frac{\varepsilon}{2} .
$$

The theorem follows from Definition 11 applied to the $T$-process and the above two inequalities.

The final construction is done by first defining a particular sequence $\left\{M_{i}\right\}$ of $M$-processes and sequence $\left\{\left(t_{i}, t_{i}^{\prime}\right)\right\}$ of pairs of points, with certain properties.

Choose a set of parameters $\left\{\lambda_{i} ; a_{i}\right\}$ such that $\lambda_{i}>0, a_{i}>0, \sum_{i=1}^{\infty} \lambda_{i}=\infty$, and $\sum_{i=1}^{\infty}\left(a_{i}\right)^{-1}<\infty$. By Theorem 3 there exist $\left(\lambda_{1}^{*}, a_{1}^{*}\right)$ finer than $\left(\lambda_{1}, a_{1}\right)$ such that the $\left(\lambda_{1}^{*}, a_{1}^{*}\right) T$-process has $\mathrm{DQ}\left(t_{1}^{\prime}, t_{1}\right) \geqq 1,\left(\varepsilon_{1}, \eta_{1}\right)$ protected for some 
$t_{1}^{\prime}>t_{1}>0, \varepsilon_{1}>0$, and $\eta_{1}>0$. By Theorem 4 there exist $\left(\lambda^{*}, a_{i}^{*}\right)$ finer than $\left(\lambda_{i}, a_{i}\right), i \geqq 2$, such that the $M$-process with parameters $\left\{\lambda_{i}^{*} ; a_{i}^{*}\right\}$ is post $\left(\varepsilon, 1, t_{1}^{\prime}\right)$ free. The $M_{1}$-process is defined by the parameters $\left\{\lambda_{i}^{*} ; a_{i}^{*}\right\}$. It will have $\mathrm{DQ}\left(t_{1}^{\prime}, t_{1}\right) \geqq 11$-fixed by Theorem 5 .

$M_{i}$ and $\left(t_{i}, t_{i}^{\prime}\right), i \geqq 2$, are defined inductively. Let the parameters of the $M_{k-1}$ process, $k \geqq 2$, be $\left\{\lambda_{i} ; a_{i}\right\}$. By Theorem 3 there exist $\left(\lambda_{k}^{*}, a_{k}^{*}\right)$ finer than $\left(\lambda_{k}, a_{k}\right)$ such that the $\left(\lambda_{k}^{*}, a_{k}^{*}\right) T$-process has $\mathrm{DQ}\left(t_{k}{ }^{\prime}, t_{k}\right) \geqq k,\left(\varepsilon_{k}, \eta_{k}\right)$ protected for some $\varepsilon_{k}>0, \eta_{k}>0, \varepsilon_{k-1} / 2>t_{k}^{\prime}>t_{k}>0$, and so that the $M$-process with parameters $\left\{\lambda_{i}^{\prime} ; a_{i}^{\prime}\right\}, \lambda_{i}^{\prime}=\lambda_{i}$ and $a_{i}^{\prime}=a_{i}, i \neq k, \lambda_{k}^{\prime}=\lambda_{k}^{*}, a_{k}^{\prime}=a_{k}^{*}$, is pre $\left(\varepsilon_{k}, k, t_{k}^{\prime}\right)$ free. By Theorem 4 there exist $\left(\lambda_{i}^{*}, a_{i}^{*}\right), i>k$, such that the $M$-process with parameters $\left\{\lambda_{i}^{*} ; a_{i}^{*}\right\}$, with $\lambda_{i}^{*}=\lambda_{i}$ and $a_{i}^{*}=a_{i}, i<k$, will be post $\left(\varepsilon_{k}, k, t_{k}^{\prime}, \eta_{k}\right)$ free. The $M_{k}-$ process is defined by the parameters $\left\{\lambda_{i}^{*} ; a_{i}^{*}\right\}$ and is $\mathrm{DQ}\left(t_{k}^{\prime}, t_{k}\right) \geqq k, k$-fixed be Theorem 5.

Now set $\left(\lambda_{i}^{(i)}, a_{i}^{(i)}\right)$ equal to the $i$ th parameters of the $M_{i}$-process, for all $i$. By the nature of the $M_{i}$, it is obvious that the parameters $\left\{\lambda_{i}{ }^{(i)} ; a_{i}^{(i)}\right\}$ define an $M$ process, and the $M$-process so defined is the desired one. For it has

$$
\frac{p_{\gamma \gamma}\left(t_{i}^{\prime}\right)-p_{\gamma \gamma}\left(t_{i}\right)}{t_{i}^{\prime}-t_{i}} \geqq i,
$$

and therefore by the mean value theorem there is a sequence of points $\left\{t_{i}^{*}\right\}$ tending to zero such that $p_{\gamma \gamma}^{\prime}\left(t_{n}^{*}\right) \geqq n$. Hence lim $\sup _{: \rightarrow 0} p_{\gamma \gamma}^{\prime}(t)=+\infty$.

\section{REFERENCES}

1. K. L. Chung, Markov chains with stationary transition probabilities, Springer-Verlag, Berlin, 1960.

UNIVERSTTY OF CALIFORNIA, Berkeley, CALIForNia 